\title{
Arriving at a correlation between the flagellar arrangement and multicellularity
}

\author{
Suruchi Jamkhedkar ${ }^{1}$, Jyotsna Dongerdive ${ }^{2}$, Kavita Jain ${ }^{2}$, Siby Abraham ${ }^{3}$, Jacinta S. D’Souza ${ }^{4 *}$ \\ ${ }^{1}$ Life Sciences Department, University of Mumbai, Kalina Campus, Mumbai, India \\ ${ }^{2}$ Computer Science Department, University of Mumbai, Kalina Campus, Mumbai, India \\ ${ }^{3}$ Mathematics and Statistics Department, G. N. Khalsa College of Arts, Science and Commerce, Mumbai, India \\ ${ }^{4}$ Department of Atomic Energy, Centre for Excellence in Basic Sciences, University of Mumbai, Kalina Campus, Mumbai, India \\ Email: *jacinta@cbs.ac.in
}

Received 4 November 2012; revised 11 December 2012; accepted 13 January 2013

\begin{abstract}
Cilia and flagella are organelles of motility that enable cells to swim or move liquid over its surface. An exhaustive literature survey for the presence of the organelle in organisms across phyla showed that most animal cells harbor cilia in contrast to very few fungal cells. While this was not unexpected, it was the position and arrangement of this organelle in each cell that intrigued our attention. Natural selection might have favored motility over chemotaxis; and it would have done so to evolve a stable structure that could have undergone an optimization process requiring a precise geometry in the shape of cells and the structure that would help cells to move. The positioning of such a structure would play a pre-dominant role in optimal motility. It is now known that the flagellar position of a cell is a genetically distinct trait, occasionally used in phylogeny of bacteria, distributed in distinguishing patterns over cellular surface, but basically are of two types, either polar (one flagellum arising from one pole per cell) or peritrichous (lateral flagella distributed over the entire cell surface). Irrespective of the cellular habitat, flagella origin, ultrastructure and proteome, the present investigation surveyed 26 sub-types of flagellar arrangements from as many species as possible. A peculiar pattern ensued-Prokaryotes harbored predominantly polar and peritrichous types; eukaryotes showed a mere change of the peritrichous one. These numbers when used to create a Similarity tree depicted a similarity distance of 14 between the Eubacteria and Archaebacteria forming the first neighborhood; Protozoans, Algae, Fungi, Plantae and Animalia formed a second neighborhood. We offer a working hypothesis for this pattern and the gradual shift in the flagellar arrangement from polar, peritrichous, sub-apical, and
\end{abstract}

"Corresponding author. apical to lateral throughout evolution.

Keywords: Flagella; Cilia; Polar; Peritrichous; Multicellularity; Flagellar Arrangement

\section{INTRODUCTION}

Most organisms modulate their motility in response to environmental variables such as nutrients, abiotic and biotic stress [1,2]. A recent study shows that, organisms probably randomized their motility in such an environment [3]. These movements range from sliding to gliding over a substrate, rotation, swimming or streaming in a suitable medium with the help of organelles such as flagella or cilia that support such motions. The once vestigial organelle, the flagellum, and its evolution is of enormous interest to biologists because the three known varieties (eukaryotic, bacterial, and archaeal flagella) represent an advanced protein-based structure that requires the interaction of its components within itself and with many systems; their molecular mechanism of motility and sensory transduction remaining elusive. An approach to the evolutionary origin of any cellular component(s) is to devise in silico homology searches among the protein or nucleotide sequences of the two components that one is trying to find evolutionary relatedness. This very approach has suggested that a subset of the bacterial flagellar components is similar to the Type III secretory and transport system; the latter defensive systems provide the function of injecting a toxin into eukaryotic cells. On the other hand, the recently elucidated archaeal flagellum has been analogous, not homologous, to the bacterial one. While there is no sequence similarity among the genes of the two systems and that the archaeal flagellum appears to grow from the base and not the tip; sequence comparison shows that the archaeal flagellum is homologous to Type IV pili [4].

The evolutionary origin of the eukaryotic flagellum 
offers two competing models; the Margulis' model disputes that the eukaryotic flagellum evolved from a symbiotic spirochete that attached to a primitive unicellular eukaryote or archaebacteria. Endogenous, autogenous and direct filiation model states that the cilium developed from pre-existing components of the eukaryotic cytoskeleton (which has tubulin and dynein) as an extension of the mitotic spindle apparatus. The first line of evidence is seen in the various early branching singlecelled eukaryotes that have a microtubule basal body, where microtubules on one end form a spindle-like cone around the nucleus, and those on the other end point away from the cell to form the cilium. A second connection is that the centrioles, homologous to the cilium, and have often been the basal body from which the cilium grows. Regarding the origin of the individual protein components, and in particular on the evolution of dyneins, the complex protein family of ciliary dynein has an apparent ancestor in a simpler cytoplasmic dynein (which itself has evolved from the AAA protein family that occurs widely in all archea, bacteria and eukaryotes). Long-standing suspicions that tubulin was homologous to FtsZ (based on very weak sequence similarity and some behavioral similarities) were confirmed by the independent resolution of the 3-D structures of the two proteins [5]. While the flagella lineage is traced back to the secretory systems of bacteria, the pili themselves is a piece of evolutionary enigma.

Notwithstanding, the flagella and not the pili are therefore, the organelles used as one of the parameters in taxonomy. Among motile rod-shaped bacteria, the number of flagella per cell, the shape of flagella and their points of origin of the cell (polar versus peritrichous), are a primary taxonomic criterion [6] with immense clinical significance [7]. Flagellar arrangement may be a feature with enough stability and uniqueness to classify flagellated bacteria into several groups [8]. Conventionally, four arrangements are popular, the monotrichous, amphitrichous, lophotrichous and polytrichous. However, variations on the classical flagellum does exist, possession of both lateral and polar flagella on the same cell, and the periplasmic flagella of spirochetes $[9,10]$. The number and position of flagellum filaments on the surface of bacterial cells are highly conserved traits, with the polar and peritrichous arrangements being frequent. Though the type of cell flagellation used as an extra taxonomic key to distinguish motile bacteria, little is known about the mechanisms regulating its the number and position [11]. The same criterion need not be used for eukaryotic species' classification. This kind of robustness, therefore, calls for the uniqueness of the flagella system across evolution. If the origin of flagella is considered, the need is to analyze the common as well as differing features in these structures throughout evolution.
An unbiased peek into the cellular position of flagella at the morphological level was attempted in the present study; with the flagella characteristics of the phyla across all the kingdoms being scored. The overall results show that the flagellar arrangement on both uni- and multicellular organisms may have evolved with a function; the position on the cell where the flagella are arranged would not have been a random selection. Whatever the purpose, our observations show a gradual shift in the arrangement of flagella from polar, peritrichous, sub-apical, apical to lateral throughout evolution; such an arrangement shifting functionally from the polar, chemo-tactically motile to the lateral, non-motile cilia types. We propose a model for multicellularity based on the arrangement of flagella; a phenomenon that is robust and reproducible from one generation to the other among all species. And, if natural selection favored motility, at least for the sake of costbenefits, arrangement of the flagella seemed critical.

\section{MATERIAL AND METHODS}

The present study is divided into two modules namely Module A and Module B.

\subsection{Module A Involves the Following Seven Knowledge Discovery Steps}

1) Taxonomic Classification.

2) Collection of Data.

3) Mapping of Flagella Information into the Taxonomical Classification.

4) Preprocessing of the Data.

5) Export of the Data to MS-SQL Server 2000.

6) Data Retrieval.

7) Data Pattern Analysis.

1) Taxonomic Classification: Based on Carl Woese Modified Classification of Robert Whittaker into six kingdoms, namely, Eubacteria, Archaebacteria, Protista, Fungi, Plantae and Animalia. The kingdoms were further classified into "Phylum", "Class", “Order", "Family", "Genus" and "Species" into a MS-Excel worksheet called Taxonomy.

2) Collection of Data: The data involved information on flagella like "Presence or Absence of Flagella", "Flagella type”, "Flagella number", "Cell Size” and "Flagella Length". The data obtained was stored in a MS-Excel worksheet called FlagellaInfo.

3) Mapping of Flagella Information into the Taxonomical Classification: The data in the Taxonomy of step 1 were mapped to FlagellaInfo of step 2. This resulted in a MS-Excel worksheet named "Flagella" with 386 records. Thus, we had the flagella information for each of the kingdoms.

4) Preprocessing of the Data: The worksheet "Flagella” was cleaned by deleting null and insignificant 
entries and segregating the data based on cell motility within the flagella type into motile and non-motile. Thus, we obtained three updated worksheets_- "Integrated Flagella" with "presence or absence of Flagella" and "Flagella type”, "MotileFlagella" and "NonMotileFlagella" based on cell motility.

5) Export of the Data to MS-SQL Server 2000: The worksheets "IntegratedFlagella”, "MotileFlagella” and "NonMotileFlagella" were exported to MS SQL server 2000 resulting in three databases called "MS-IntegratedFlagella”, "MS-MotileFlagella” and "MS-NonMotileFlagella” respectively for further processing.

6) Data Retrieval: SQL queries were fired on these databases so as to analyze the distribution of various types of flagella present in the kingdom, Phyla with and without flagella present in each of them, etc.

Select count $(*)$ from New Database where Phylum = "Actinobacteria" and Kingdom = "Bacteria".

Select count $(*)$ from NewDatabase where Phylum = "Crenarchaeota" and Kingdom = "Archeabacter".

Select count $(*)$ from NewDatabase where Phylum = "Heterokontophyta" and Kingdom = "Protista".

7) Data Pattern Analysis: The query results obtained in step 6 were represented as bar diagrams for analyzing the pattern emerged from the data.

\subsection{Module B Involves the Construction of Dendogram}

The current study offers a novel methodology for the construction of a DendoGram which reflect the phenotypic similarities between different operational taxonomic units (OUT). The methodology is inspired by UPGMA Method (Unweighted Pair Group Method with Arithmetic Mean) [12] used for constructing a Similarity tree. However, the procedure takes the liberty of not using DNA or Protein sequences which is conventional for the construction of similarity trees. The procedure uses morphology of 26 Flagella types/arrangements present across 6 kingdoms. This is realized by represen- ting "Presence" of "Flagella type" by "P” and “Absence" of "Flagella type" by "A". The result is a sequence of 6 strings each having length 26 wherein each individual string is treated as an OTU.

Since the flagella types are different in Prokaryotes and Eukaryotes, the OTUs were classified into two mutually exclusive sets as given below:

$\mathrm{X}=\{$ Eubacteria, Archaebacteria $\}$.

$\mathrm{Y}=\{$ Protista, Fungi, Plantae, Animalia $\}$ as given in Tables 1 and 2.

The method involved the construction of distance matrix, the entries of which, are calculated by comparing an OTU with other OTUs and the number of identities was taken as the score of that OTU. This resulted in a distance matrix for strings given in Table 2 as shown below:

\begin{tabular}{cccccc}
\hline & C & D & E & F & G \\
\hline C & 0 & & & & \\
D & 1 & 0 & & & \\
E & 2 & 1 & 0 & & \\
F & 2 & 1 & 0 & 0 & \\
G & 8 & 7 & 6 & 6 & 0 \\
\hline
\end{tabular}

The two OTUs having the minimum value in the distance matrix was treated as a new single "composite" OTU which became the initial nodes of the tree. A new distance matrix is constructed with the composite and remaining single OTUs. This process continued until only two OTUs were left. This resulted in the formation of a dendogram which showed the local homology between different kingdoms. The findings on the evolution of flagella based on their arrangements were drawn by analyzing the bar diagrams and the dendogram constructed.

\section{RESULTS AND DISCUSSION}

The first primitive cell, assumed as a non-motile coccus

Table 1. OTU of prokaryotes.

\begin{tabular}{|c|c|c|c|c|c|c|c|c|c|c|c|c|c|c|c|c|c|c|c|c|c|c|c|c|c|c|}
\hline Eubacteria (B) & $\mathrm{P}$ & $\mathrm{P}$ & $\mathrm{P}$ & $\mathrm{P}$ & $\mathrm{P}$ & $\mathrm{P}$ & $\mathrm{P}$ & $\mathrm{P}$ & $\mathrm{P}$ & $\mathrm{P}$ & $\mathrm{P}$ & A & A & A & A & A & A & A & A & A & A & A & A & A & A & A \\
\hline Archaebacter (R) & $\mathrm{P}$ & $\mathrm{P}$ & A & $\mathrm{P}$ & A & A & $\mathrm{P}$ & A & $\mathrm{P}$ & A & A & $\mathrm{P}$ & $\mathrm{P}$ & $\mathrm{P}$ & $\mathrm{P}$ & $\mathrm{P}$ & $\mathrm{P}$ & $\mathrm{P}$ & $\mathrm{P}$ & A & A & A & A & A & A & A \\
\hline
\end{tabular}

Table 2. OTU of eukaryotes.

\begin{tabular}{|c|c|c|c|c|c|c|c|c|c|c|c|c|c|c|c|c|c|c|c|c|c|c|c|c|c|c|}
\hline Protista (C) & A & A & A & A & A & A & A & $\mathrm{P}$ & A & A & A & A & A & A & A & A & A & A & A & $\mathrm{P}$ & $\mathrm{P}$ & $\mathrm{P}$ & $\mathrm{P}$ & A & A & A \\
\hline Fungi (D) & A & A & A & A & A & A & A & $\mathrm{P}$ & A & A & A & A & A & A & A & A & A & A & A & $\mathrm{P}$ & $\mathrm{P}$ & A & A & A & A & A \\
\hline Plantae (E) & A & A & A & A & A & A & A & $\mathrm{P}$ & A & A & A & A & A & A & A & A & A & A & A & $\mathrm{P}$ & $\mathrm{P}$ & A & A & A & A & A \\
\hline Animalia (F) & A & A & A & A & A & A & A & A & A & A & A & A & A & A & A & A & A & A & A & A & A & A & A & $\mathrm{P}$ & $\mathrm{P}$ & $\mathrm{P}$ \\
\hline
\end{tabular}


was probably carried by currents present in the primordial milieu. From here on, two forms of movements were notably seen; cells with gas vesicles and those that harbored flagella. Gas vesicles are found rampantly among the unicellular organisms; but, flagella are found equally among both the unicellular and multicellular organisms. The latter might have originated from groups of single cells that need to show high interaction and co-operation between one another and be nearly free of variance [13]. The evolution of flagella has always been a curiosity to biologists, particularly in the context of non-similarities in the ultrastructure, development and motion dynamics of the three known varieties (viz. Eukaryotic, bacterial, and the archaeal types) [14-17]. Some overlaps in the gene/protein sequence similarities have led many to believe that the eubacterial flagella originated from the T3SS, while the archebacterial flagella originated from the T4SS [14-17]. With two competing views on the evolutionary origin of the eukaryotic flagellum, the one that seems largely favored in terms of gene/protein sequence similarity is the endogenous or direct filiation model. The pre-existing components of the eukaryotic cytoskeleton such as tubulin and dynein are extensions of the mitotic spindle apparatus. Dynein evolution shows that the complex protein family of ciliary dynein has evolved from a simpler cytoplasmic dynein that evolved from the AAA protein family that occurs widely in all archaea, bacteria and eukaryotes [15]. These support, mainly vertical inheritance for the components of this unique motility system, and probably an ancient horizontal gene transfer event of a whole flagellum-coding gene cluster among Euryarchaeota and Crenarchaeota [18]. In the context of all these sequence similarities and an associated functional significance of cilia in motility, sensory transduction and development, we set out to know whether there an order or randomness in the rather simple attribute, namely, flagellar arrangement of cells across all the six kingdoms? And, could this arrangement serve as a paradigm to draw a similarity tree, akin to a phylogenetic tree so as to reveal the relatedness between species, if any?

The study, initiated by surveying the flagella literature (290 primary references, and references therein amounting to $\sim 6000$ ) for flagella presence with respect to the arrangement; irrespective of their internal ultrastructure (flagellin-based or tubulin-based), number, length and functionality (motile, non-motile or sensory) of the organelle on the cell. The comparison and purpose behind the work are entirely based on the common features in their physical properties and functions across species; this being, property of locomotion, sensory perception, made up of protein polymers, have ATPase or proton-motive forces, anchored to a basal body, sizes are about $2 \mathrm{~mm}$ - $40 \mathrm{~mm}$, complex and made up of several proteins. In order to avoid the data being skewed, flagella information from 357 species (Eubacteria-115, Archaebacteria-129, Protista-37, Fungi-19, Plantae-19 and Animalia-38) are provided in this study. Such a survey led to a database for the presence or absence of the organelle, and its arrangement vis-à-vis the species classified using the classical method of taxonomical classification. As detailed in the methods, the database was queried and the percentage of species that have flagella within each phylum calculated. The percentages were calculated by the number of phyla having flagella verses total phyla in that particular kingdom. As depicted in Figure 1, the percentages of organisms (multicellular and/or unicellular) harboring or not harboring flagella varied with the phyla among all the kingdoms. For example, $80 \%$ of the phyla among Eubacteria have flagella, while only $20 \%$ of phyla in Archaebacteria showed flagella. In Protista, 77\% phyla have flagella while in Fungi $10 \%$ phyla have flagella. In Plantae, $70 \%$ phyla have flagella. All the phyla (100\%) in the Animalia kingdom have flagella or cilia. A noteworthy comment is that the flagella in the Fungi and Plantae kingdoms belong to the cells of the reproductive system (zoöspores).

Having depicted the gross variability in the presence and/or absence of flagella among different kingdoms, the flagellar arrangement of the cells was then categorized. Detailed observation of images of cells bearing flagella helped in making twenty-four categories of flagellar arrangements (Figure 2(a)). While, twenty-four arrangements of flagella were totally observed; any other arrangement considered "cryptic"; the latter type being the most prevalent ( 23\%; Figure 2(b)). Among the known categories, the predominating flagellar arrangements were of the polar type ( 15\%) followed by peritrichous $(\sim 11 \%)$ and lateral ( 10\%; Figure 2(b)). However, the other types of arrangements (multilateral, monopolar polytrichous, multitrichous, monotrichous, polar multiple

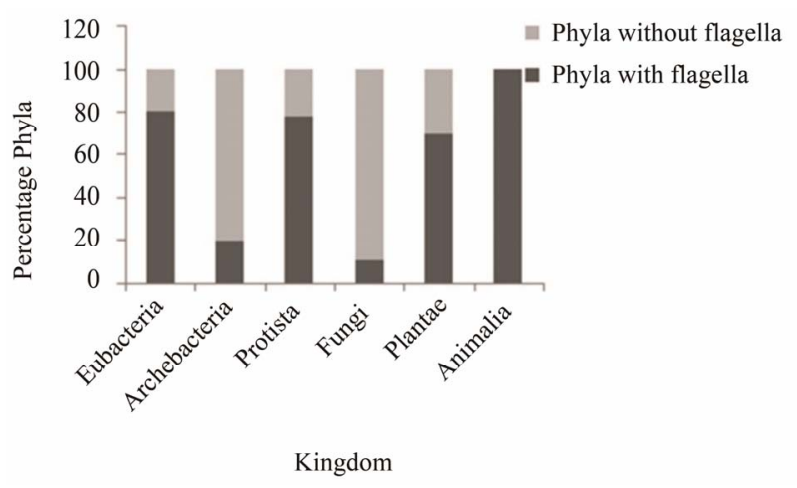

Figure 1. Presence or absence of flagella in various kingdoms. Phyla with and without flagella were scored and a bar diagram showing the percentage of phyla with or without flagella across the six kingdoms were obtained. 


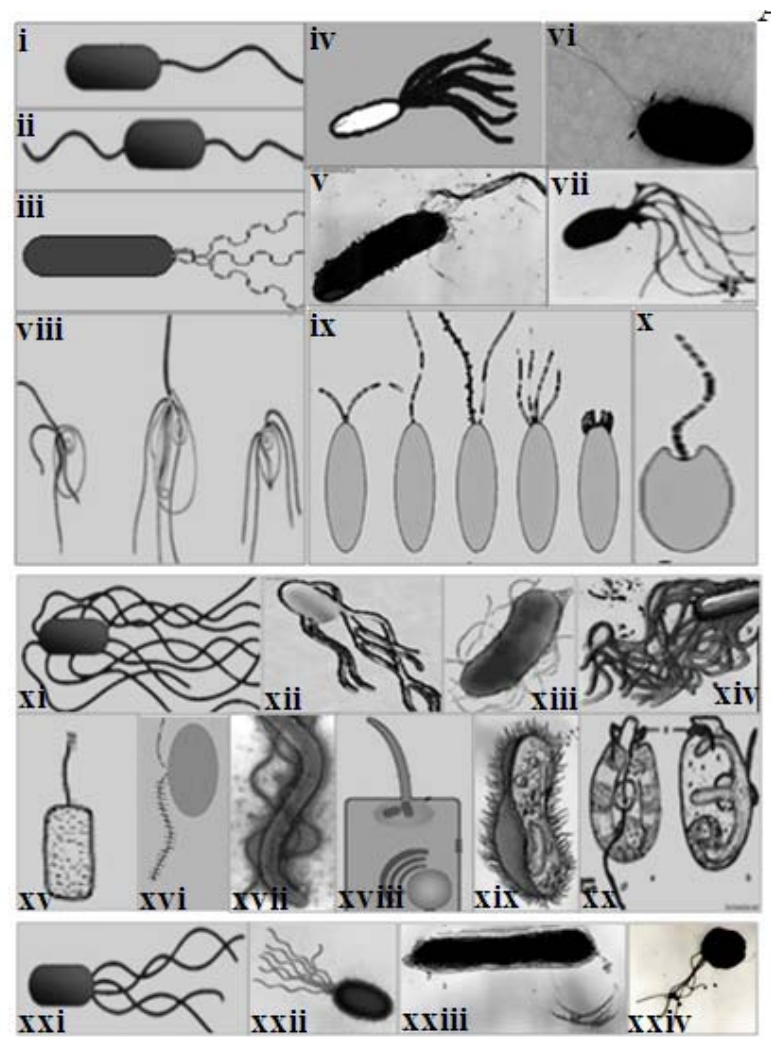

(a)

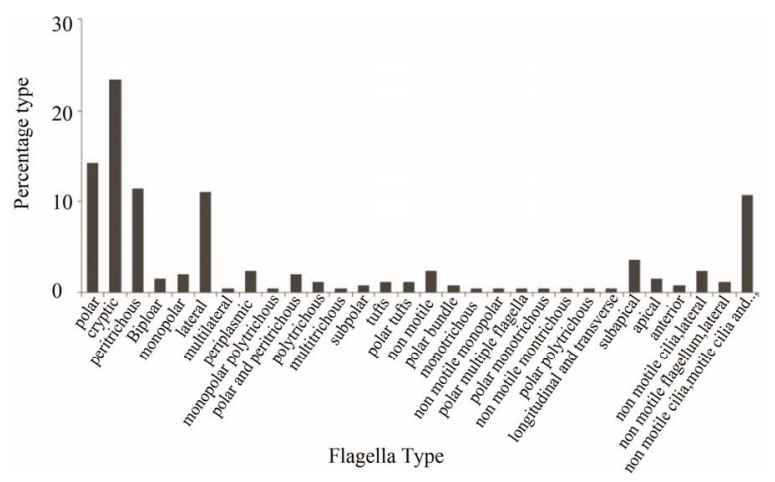

(b)

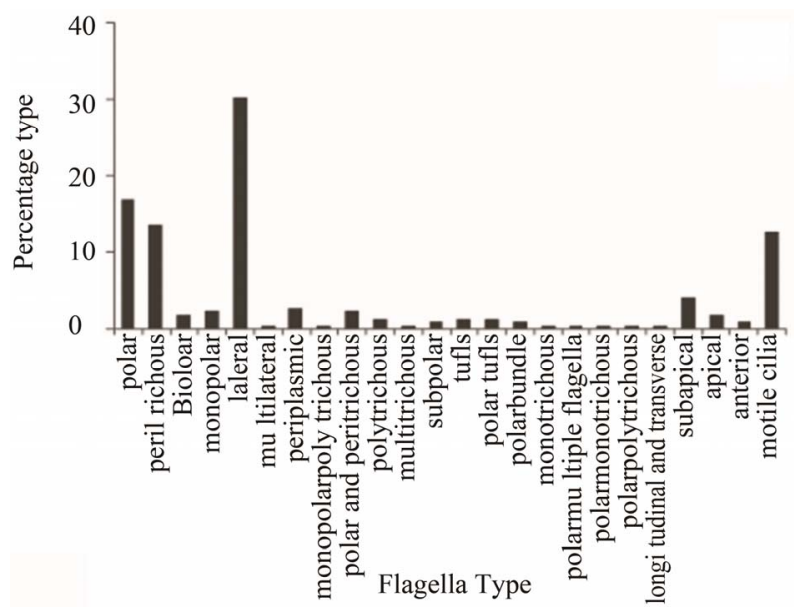

(c)

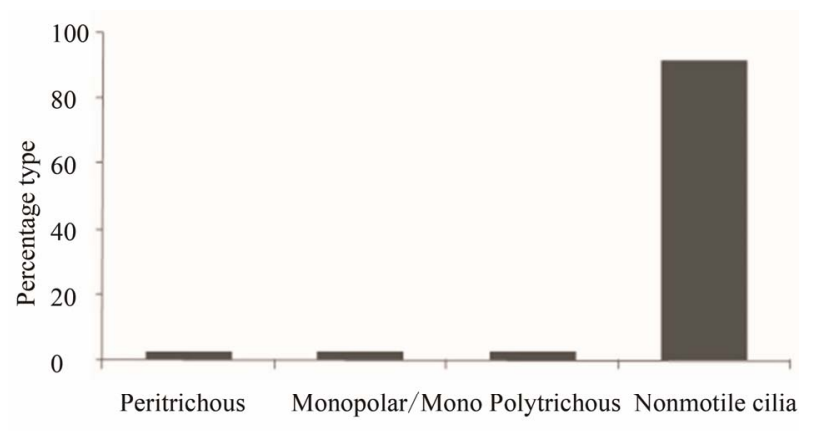

Flagella Type

(d)

Figure 2. (a) Flagella types and their variations as depicted in the literature. A pictorial (cartoon, 2D) summary of the flagella is depicted into three types. Note that the Polar (panels i to $\mathrm{x}$ ) and Peritrichous (panels xi to $\mathrm{xx}$ ) are considered as the major types and the variations therein are depicted as (i) Polar, (ii) Bipolar, (iii) Monopolar, (iv) Polar Tufts, (v) Polar Bundle, (vi) Subpolar, (vii) Tufts, (viii) Anterior, (ix) Apical, (x) Sub Apical, (xi) Peritrichous, (xii) Polytrichous, (xiii) Multitrichous, (xiv) Multilateral, (xv) Monotrichous, (xvi) Lateral, (xvii) Periplasmic, (xviii) Cilia, (xix) Motile Cilia, and (xx) Longitudinal Transverse. Those flagellar types that could not be described as either polar or peritrichous were categorized as cryptic (panels xxi to xxiv) (xxi, Polar Peritrichous; xxii, Polar Multiple; xxiii, Monopolar Polytrichous and xxiv, Polar Polytrichous); (b) Arrangements of Flagella present across all kingdoms. The histograms show the percentages of various flagella types; irrespective of their structure, proteome and function, present across six kingdoms obtained by taking the different flagella types depicted in (a) and its occurrence in percentage along Y-axis; (c) Diverse motile flagella types and their occurrence. Among the arrangements scored in (b) the quantitative occurrence of percentage motile flagella types was plotted along $\mathrm{X}$-axis and corresponding percentage along Y-axis; (d) Diverse Non-Motile Flagella type and its occurrence. Bar diagram showing occurrence of different non-motile flagella types in percentage obtained by plotting different non-motile flagella types versus corresponding percentage along $\mathrm{Y}$-axis.

flagella, non motile monopolar, non motile monotrichous, polar monotrichous, polar polytrichous, longitudinal and transverse, subpolar, polar bundle, anterior, polytrichous, tufts, polar tufts, non motile flagella lateral, bipolar, apical, monopolar, polar and peritrichous, periplasmic, non motile, non motile cilia lateral, sub apical and non motile cilia, motile cilia, lateral) varied from $0.4 \%$ - $10 \%$ and were mere variations of the polar and peritrichous types (Figure 2(b)). Since flagella and/or cilia have an associated function of motility and therefore, a correlation between flagellar arrangements vis-à-vis motility sought (Figure 2(c)). Among all the motile flagella/cilia, the lateral (30\%) arrangement was the most prevailing followed by polar (16\%), peritrichous (14\%) and secondary cilia (13\%; Figure 2(c)). Flagellar arrange- 
ment diversity among motile organisms was vast; although the total number of organisms harboring such a variety ranged between $0.5 \%$ - $5 \%$ only. On the other hand, among the non-motile flagella/cilia, the primary cilia (seen in the Animalia kingdom) are the most widespread, followed by the peritrichous, mono- and polytrichous categories (Figure 2(d)). Therefore, the first observation remains that the flagella which offer motility are predominately polar and peritrichous in nature and are prevalent among the Eubacteria (Figure 2(c)); the lateral flagella being found in fungi, plants and animals. Meanwhile, the flagella on non-motile cells are mainly found in Archaebacteria and Animalia kingdoms, predominate being mono- or poly- trichous group (Archaebacteria) (Figure 2(d)).

To gain an evolutionary perspective, the organisms harboring flagella from various kingdoms were separately scored for their flagellar arrangements (Figures 3(a)-(f)). These results show that flagellar arrangement diversity between the prokaryotes is higher ( 13 in numbers) than that of eukaryotes (between 3 - 5). Nonetheless, the main flagellar arrangement remains of the polar and peritrichous types in prokaryotes and lateral among all the eukaryotes. In the Eubacteria kingdom, the polar flagella (34\%) group predominates, followed by the peritrichous (28\%) arrangement (Figure 3(a)). As observed, the peritrichous flagella are mainly seen in the pathogenic species of Eubacteria kingdom [19]. This further supports the idea put forth by Jensen in 1909 that the most primitive form of life would have been cells with polar flagella and in accordance, ancestors of prokaryotes [20,21]. Also, flagella conversion from one form to another is reported only among prokaryotes [8-10,22]. Incidentally, certain bacteria when grown at a higher temperature shed their flagella [23]. We observed that most of the organisms in Archaebacteria are without flagella (Figure 1). And among these 20\% which harbor flagella, fifteen arrangements with the polar and peritrichous as widespread (Figure $\mathbf{3 ( b )}$ ). Those which have flagella are further classified based on cell motility. The flagella of non-motile cells have predominately peritrichous flagella. This flagella type is thinner than the peritrichous flagella seen in motile cells [24,25]. The flagella of motile cells are mainly polar type. As observed in Archaebacteria, similarly, most species in Protista kingdom are without flagella (Figure 1). In contrast, those that have flagella are of lateral, apical, sub-apical, anterior or longitudinal transverse types; the sub-apical (39\%) is of the major type, followed by lateral (33\%; Figure 3(c)). It is also observed that liverworts have unequal length flagella. In these unequal flagella, the shorter one is tensile and the longer one has the whiplash kind of movement [26]. From among the fungal species, the flagella are mainly found on the zoöspores and these are the lateral, apical and sub-apical types (Figure 3(d)). Except for some unicellular algal cells, the dominant type of flagella is lateral in the Plantae kingdom, (Figure 3(e)), and these are present on zoöspores. It is noteworthy that the apical and sub-apical type of flagellar arrangement is particularly found among lower marine plants such as seaweed and sea lettuce [27]. Along with the flagella, cilia are also observed throughout the Animalia kingdom; these are both motile and non-motile. The flagella are mainly on sperms, and the position is predominantly lateral (Figure 3(f)). In summary, if one considers only two major types of arrangements of flagella across kingdoms, and then looks closely at these types and their variations, one observes that variations of polar and peritrichous types are mostly among the bacterial species and not among the eukaryotes (Table 3). A clear transition from the polar, peritrichous to the only peritrichous types across evolution is markedly clear.

With the flagellar arrangement into consideration, a Dendogram (Figure 4) was constructed based on "flagella types" whose values were represented as "P” and “ $A$ ” indicating presence or absence of flagella type respectively. During this process, differences between the Prokaryotic and Eukaryotic flagella types were observed to be stark and hence were segregated as two different classes. To get an in-depth insight of eukaroytic flagellar evolution, the protista and algal kingdoms were separated. The resultant Dendogram (Figure 4) gave an insight of organismal evolution based on the flagella types. Contrary to expectations, the tree depicts similarity between the Eubacteria and Archaebacteria with a similarity distance of 14 forming the first neighborhood. Protista, Algae, Fungi, Plantae, Animalia form a second neighborhood. Protista and Algae are more similar to one another and are joined at a similarity distance of 1 . The composite taxon (1) Protista and algae is similar to Fungi at a similarity distance of 1.5 and hence forms the next composite taxon (2). The taxon (2) is similar to Plantae at a similarity distance of 1 forming taxon (3), while taxon (3) is similar to Animalia at a similarity distance of 6.75 . The distinct arrival of two neighborhoods may be interpreted as the two distinct types of flagellar ultrastructures and proteomes in the prokaryotes and eukaryotes, respectively.

In conclusion, our hypothesis restricts to the understanding of flagellar position on prokaryotes in contrast to eukaryotes; this, because work on the molecular understanding about the flagella position on prokaryotes is well understood contrary to that in eukaryotes. Our working model (Figure 5) states that the primitive cell may have been non-motile coccus, with the propensity to form the Mycoplasmas or a rod-shaped bacterium. Suitable conditions, unknown until now, may have converted this bacterium into the motile, polar cell. Flagellum 

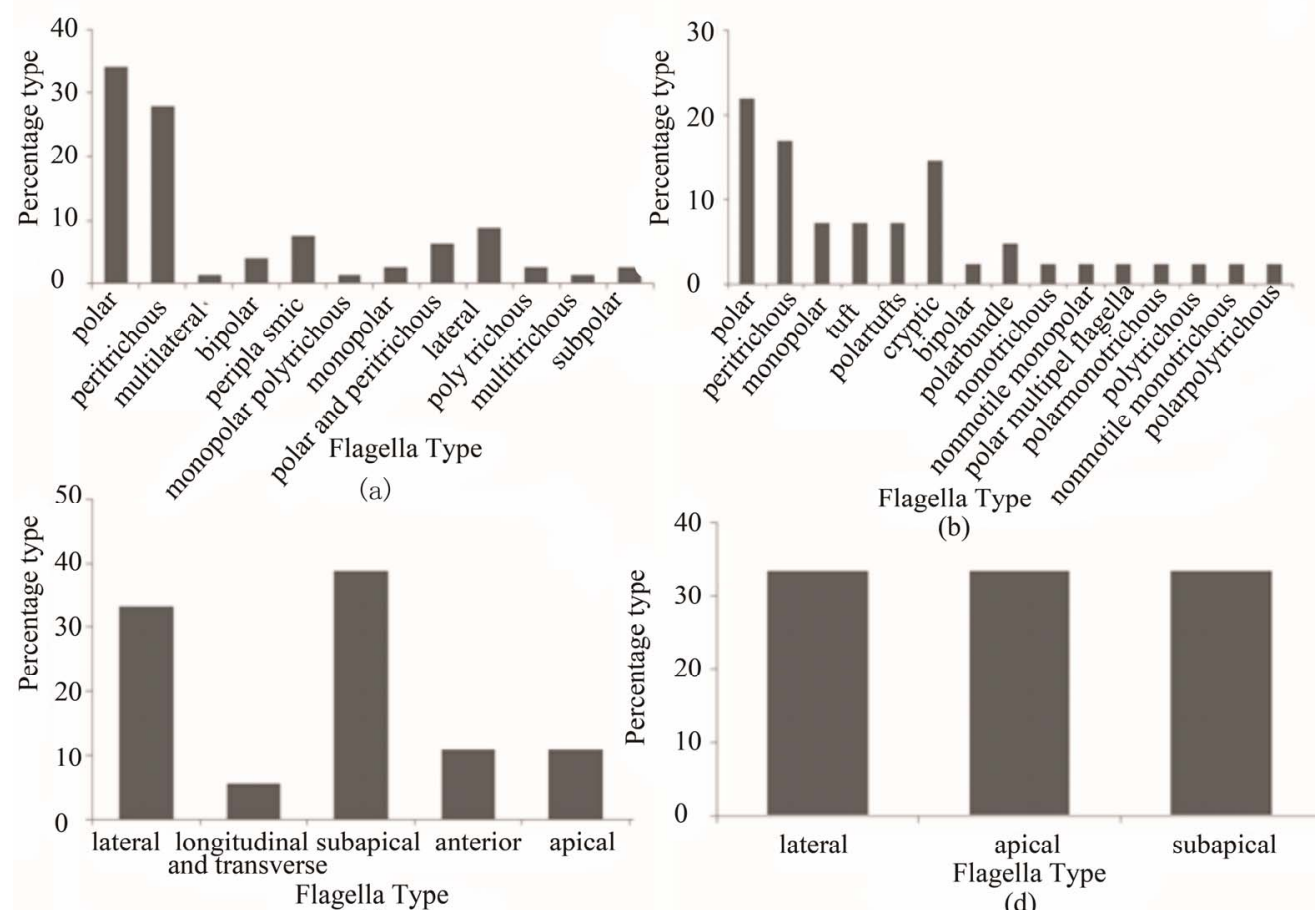

(c)

(d)

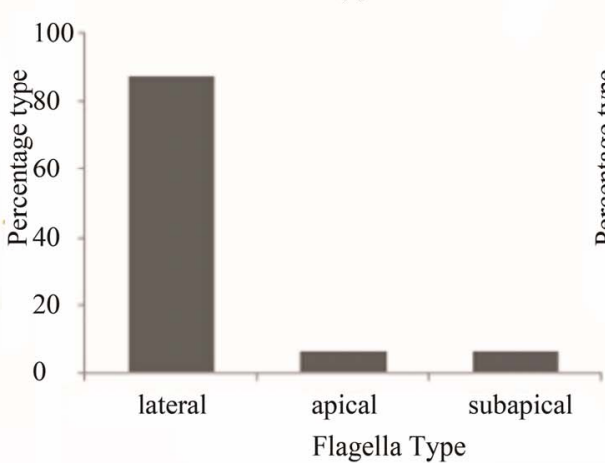

(e)

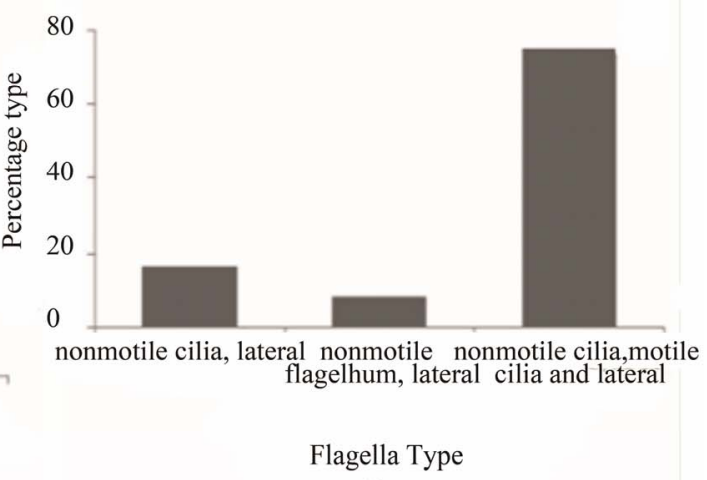

(f)

Figure 3. Flagella types among various Phyla. Bar diagram showing occurrence of different flagella types in the Kingdom Eubacteria (a); Archaebacteria (b); Protista (c); Fungi (d); Plants (e) and Animals (f) obtained by plotting different flagella types and corresponding percentage occurrence along Y-axis.

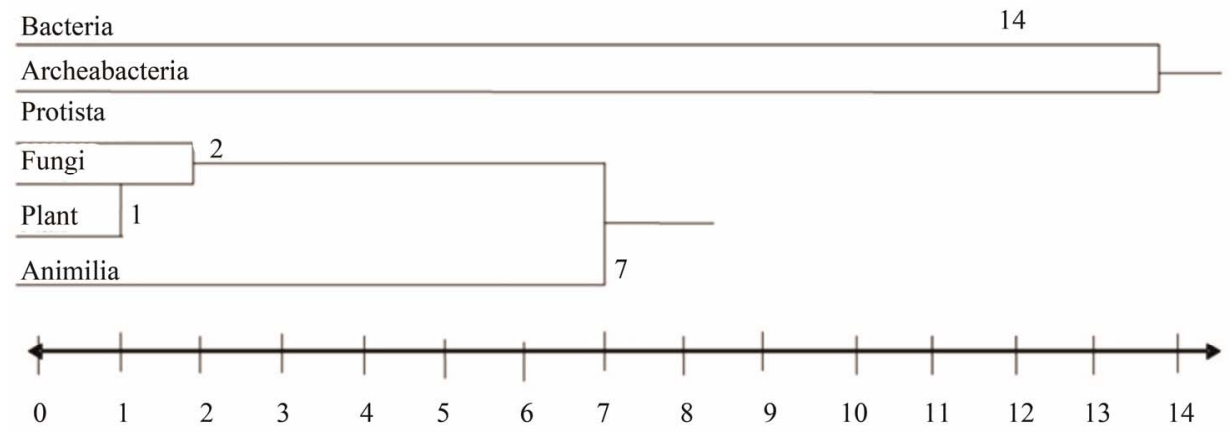

Figure 4. Similarity tree. Shows a Similarity tree is "on its side” and that the individual Kingdoms are joined using the average matching coefficients. The tree indicates that the nearest neighbour of bacteria is Archebacter, and vice versa, and that the nearest neighbour of protista is Algae, and vice versa (1 average coefficient value in both cases). Each Kingdom is located in its own leaf node in the tree. 


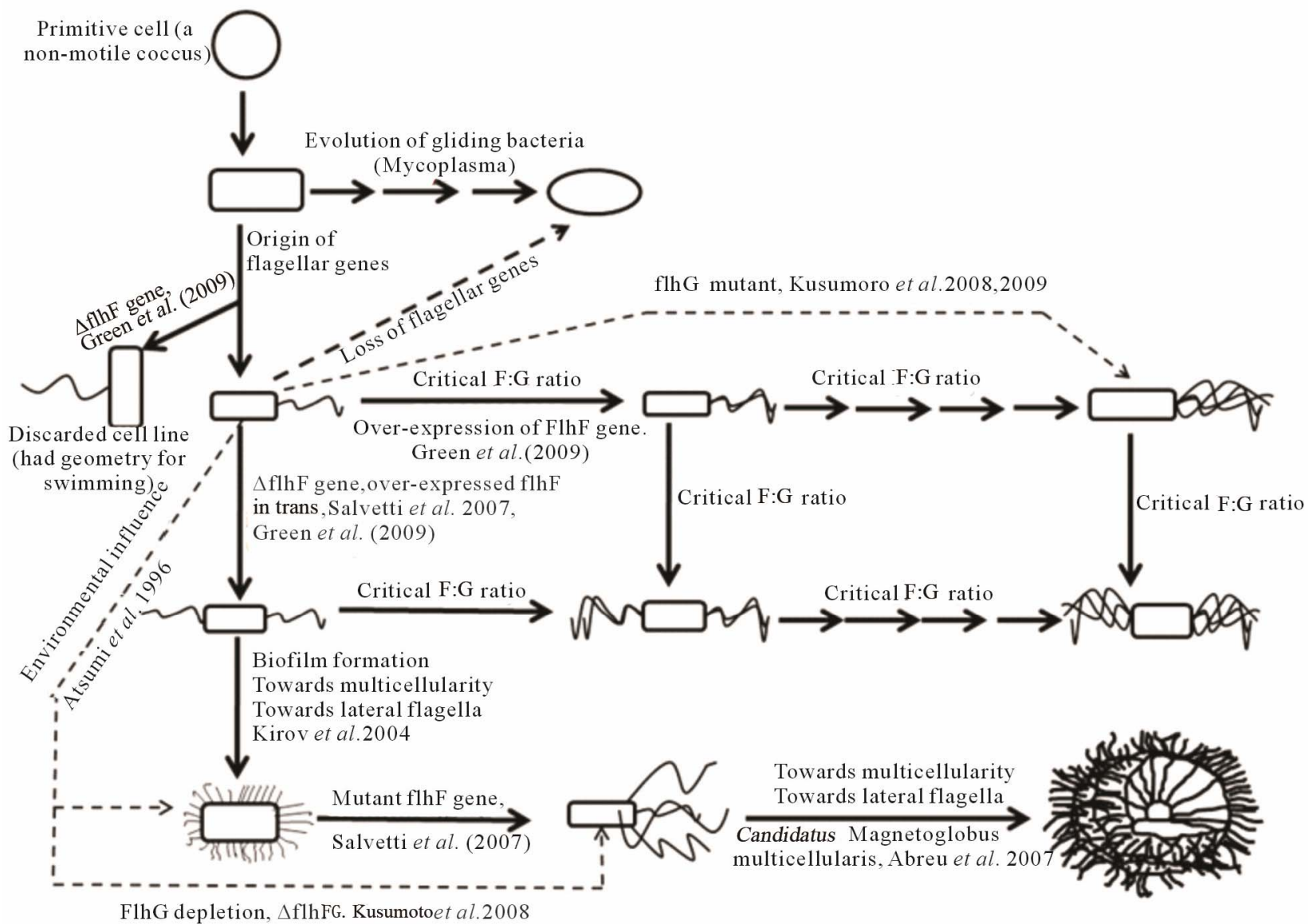

Figure 5. Working hypothesis providing in-depth analysis of the variations in the arrangements of flagella in bacterial species. Wherever possible, evidence in the form of references has been provided. As no experimental inputs or retrospective evidence was found throughout the literature for eukaryotic flagellar arrangement, the same has been largely held in reserve from this model.

Table 3. Flagellar and cellular parameters across evolution. Summary of various flagellar parameters used to compare across evolution. Note the increase in flagellation, motility, laterality and multicellularity with evolution.

\begin{tabular}{|c|c|c|c|c|c|c|}
\hline \multicolumn{7}{|c|}{ EVवपण्सू } \\
\hline Kingdom & Archaebacteria & Eubacteria & Protista & Fungi & Plants & Animals \\
\hline$\%$ Flagella & 20 & 80 & 78 & 11 & 70 & 100 \\
\hline \% Motile & 83 & 100 & 100 & 100 & 100 & 75 \\
\hline $\begin{array}{c}\text { 1st Predominant } \\
\text { arrangement }\end{array}$ & Polar & Polar & Sub-apical & $\begin{array}{l}\text { Lateral, Apical, } \\
\text { Sub-apical }\end{array}$ & Lateral & Lateral \\
\hline $\begin{array}{l}\text { 2nd Predominant } \\
\text { arrangement }\end{array}$ & Peritrichous & Peritrichous & Lateral & $\begin{array}{l}\text { Lateral, Apical, } \\
\text { Sub-apical }\end{array}$ & $\begin{array}{c}\text { Apical, } \\
\text { Sub-apical }\end{array}$ & $\begin{array}{c}\text { Primary, Non } \\
\text { motile cilia }\end{array}$ \\
\hline Origin & TIVSS & TIIISS & \multicolumn{4}{|c|}{ Cytoplasmic proteins such as AAA family and FtsZ } \\
\hline Flagella type & Flagellin & Flagellin & Tubulin & Tubulin & Tubulin & Tubulin \\
\hline Biofilm formation & Almost all & Almost all & Some & Some & Almost none & Almost none \\
\hline Cellularity & Unicellular & Unicellular & Unicellular/Multicellular & Unicellular/Multicellular & Unicellular/Multicellular & Multicellular \\
\hline
\end{tabular}


position on such a bacterium would be decided by energy costs, the geometry of the cell versus placement of the flagellum and simultaneous origin of the genes responsible for deciding flagellum position on the cell; a wrong geometry would be a misfit and eventually be discarded. The gene responsible for the position and number of flagella per cell is guanosine 5c-triphosphate (GTP) -binding protein (FlhF); it correctly localizes the flagella in several bacterial species [11,28-31]. Furthermore, an interaction between the FlhF and another protein FlhG regulates the number of polar flagella in $V$. alginolyticus cells. FlhF, localized at the pole probably promotes the flagella assembly at the initiation point, namely, the MS ring. FlhG, a cytoplasmic protein that harbors a ras-like GTPase interacts with FlhF and inhibits its localization at the pole, thus repressing excessive flagellation [29-31]. On the other hand, a cell over-expressing FlhG protein, has no FlhF molecules to localize at the pole, thus failing to produce a polar flagellum. With no FlhG, most of the FlhF localizes at the pole, such cells producing multiple flagella or tufts at the pole. We conclude therefore, that a critical ratio of FlhF and FlhG protein expression eventually leads to variations in the flagella; and mutations within these genes leading to higher or lower ratios are essentially speciation events throughout evolution. Although homologs of FlhF and FlhG are found in eukaryotes, their role in flagella position and the number is something to our knowledge not been investigated. Hence, although energy costs of locomotion are small for bacteria, fluid mechanical forces in its environment (namely, the primordial soup) may have played a significant role during the evolution of the flagellum [32]. On the lines of changing the arrangements on the cell, it seems that stress plays a vital role. In contemporary bacteria, motility being associated with chemotaxis; the response to signals from the environment modulates the motion within the cell harboring the flagella. Such changes within the environment, associated with stress have shown that bacteria can change its flagellar arrangement from one form to the other [9,10,22], causing biofilms to form that again requires flagella help. However, a recent finding indicates that motility may have evolved before chemotaxis or favored without chemotaxis [3]; flagellar arrangement for the sole function of motility is over scored among bacteria in which they are predominantly polar to peritrichous. A curiously strange phenomenon seen in the organism, Candidatus Magnetoglobus multicellularis, a multicellular, magnetotactic prokaryote from a hypersaline environment is that, it forms structures with peritrichous flagella-a signature that reflects multicellularity in terms of our working hypothesis [33]. We, therefore, attribute a changing environment (stressful niches) that may regulate the levels of FlhF and/or FlhG genes, an added function of signal transduction and multicellularity as the chief factors responsible for the evolving arrangements in the flagella of cells from polar, peritrichous, sub-apical, apical to lateral throughout evolution. Analogously, eukaryotes with the classical " $9+2$ " flagella/cilia arrangement also show changes from anterior flagella (Chlamydomonas reinhardtii) to the peritrichous form (Volvox carteri); this being a testable hypothesis [34].

\section{ACKNOWLEDGEMENTS}

Financial support to JSD was provided by the UM-DAE CBS, India.

\section{REFERENCES}

[1] Dusenbery, D.B. (1992) Sensory ecology: How organisms acquire and respond to information. Freeman, New York.

[2] Thor, R. and Kühl, M. (2003) Bacteria are not too small for spatial sensing of chemical gradients: An experimental evidence. Proceedings of the National Academy of Sciences of the United States, 100, 5748-5753. doi:10.1073/pnas.1030795100

[3] Wei, Y., Wang, X., Liu, J., Nememan, I., Singh, A.H., Weiss, H. and Levin, B.R. (2011) The population dynamics of bacteria in physically structured habitats and the adaptive virtue of random motility. Proceedings of the National Academy of Sciences USA, 108, 4047-4052. doi:10.1073/pnas.1013499108

[4] Thomas, N.A., Bardy, S.L. and Jarrell, K.F. (2001) The archaeal flagellum: A different kind of prokaryotic motility structure. FEMS Microbiology Review, 25, 147-174. doi:10.1111/j.1574-6976.2001.tb00575.x

[5] Margolin, W., Wang R. and Kumar M. (1996) Isolation of an ftsZ Homolog from the Archaebacterium Halobacterium salinarium: Implications for the evolution of FtsZ and Tubulin. Journal of Bacteriology, 178, 1320-1327.

[6] Stanier, R.Y., Doudoroff, M. and Adelberg, E.A. (1970) The microbial world. 3rd Edition, Prentice-Hall Inc., Englewood Cliffs.

[7] Hynes, M. (1968) Medical microbiology. 9th Edition, Churchill, London, 152-184.

[8] Leifson, E. (1951) Staining, Shape, and Arrangement of Bacterial Flagella. Journal of Bacteriology, 62, 377-389.

[9] Kawagishi, I., Maekawa, Y., Atsumi, T., Homma, M. and Imae, Y. (1995) Isolation of the polar and lateral flagellum-defective mutants in Vibrio alginolyticus and identification of their Flagellar Driving Energy Sources. Journal of Bacteriology, 177, 5158-5160.

[10] Atsumi, T., Maekawa, Y., Yamada, T., Kawagishi, I., Imae, Y. and Homma, M. (1996) Effect of viscosity on swimming by the lateral and polar flagella of Vibrio alginolyticus. Journal of Bacteriology, 178, 5024-5026.

[11] Salvetti, S., Ghelardi, E., Celandroni, F., Ceragioli, M., Giannessi, F. and Senesi, S. (2007) FlhF, a signal recognition particle-like GTPase, is involved in the regulation of flagellar arrangement, motility behaviour and protein 
secretion in Bacillus cereus. Microbiology, 153, 25412552. doi:10.1099/mic.0.2006/005553-0

[12] Sneath, P.H.A and Sokal, R.R. (1973) Numerical taxonomy. W.H. Freeman and Company, San Francisco, 230-234.

[13] Queller, D.C. and Strassmann, J.E. (2009) Beyond society: The evolution of organismality. Philosophical Transactions of the Royal Society B, 364, 3143-3155. doi:10.1098/rstb.2009.0095

[14] Wong, T., Amidi, A., Dodds, A., Siddiqi, S., Wang, J., Yep, T., Tamang, D.G. and Saier, M.H. (2007) Evolution of the bacterial flagellum: Cumulative evidence indicates that flagella developed as modular systems, with many components deriving from other systems. Microbe, 2, 335-340.

[15] Hall, J.L., Ramanis, Z. and Luck, D.J. (1989) Basal body/ centriolar DNA: Molecular genetic studies in Chlamydomonas. Cell, 59, 121-132. doi:10.1016/0092-8674(89)90875-1

[16] Pallen M.J. and Matzke N.J. (2006) From the origin of species to the origin of bacterial flagella. Nature Reviews. Microbiology, 4, 784-790. doi:10.1038/nrmicro1493

[17] Margulis, L. (1981) Symbiosis in cell evolution: Life and its environment on the early earth. W. H. Freeman, San Francisco.

[18] Desmond, E., Brochier-Armanet, C. and Gribaldo, S. (2007) Phylogenomics of the archaeal flagellum: Rare horizontal gene transfer in a unique motility structure. BMC Evolutionary Biology, 7, 106. doi:10.1186/1471-2148-7-106

[19] Kirov, S.M. (2003) Bacteria that express lateral flagella enable dissection of the multifunctional roles of flagella in pathogenesis. FEMS Microbiology Letters, 224, 151159. doi:10.1016/S0378-1097(03)00445-2

[20] Jensen, O. (1909) Die hauptlinien des naturlichen bakterien-systems. Centralblatt fur Bakteriologie, Section 2, XXII, 305-346.

[21] Breed, R.S., Conn, H.J. and Baker, J.C. (1918) Comments on the evolution and classification of bacteria. Journal of Bacteriology, 3, 445-459.

[22] Kirov, S.M., Castrisios, M. and Shaw, J.G. (2004) Aeromonas flagella (polar and lateral) are enterocyte adhesins that contribute to biofilm formation on surfaces. Infection and Immunity, 72, 1939-1945. doi:10.1128/IAI.72.4.1939-1945.2004

[23] Quadling, C. and Stocker, B.A. (1962) An environmenttally-induced transition from the flagellated to the nonflagellated state in Salmonella typhimurium: The fate of parental flagella at cell division. Journal of General Microbiology, 28, 257-270. doi:10.1099/00221287-28-2-257

[24] Takai, K., Nealson, K.H. and Horikoshi, K. (2004) Methanotorris formicicus sp. nov., a novel extremely thermophilic, methane-producing archaeon isolated from a black smoker chimney in the Central Indian Ridge. International Journal of Systematic and Evolutionary Microbiology, 54, 1095-1100. doi:10.1099/ijs.0.02887-0

[25] Singh, N, Kendall, MM, Liu, Y. and Boone, DR. (2005) Isolation and characterization of methylotrophic methanogens from anoxic marine sediments in Skan Bay, Alaska: Description of Methanococcoides alaskense sp. nov., and emended description of Methanosarcinabaltica. International Journal of Systematic and Evolutionary Microbiology, 55, 2531-2538. doi:10.1099/ijs.0.63886-0

[26] Renzaglia, K.S. and Duckett, J.G. (1991) Towards an understanding of the differences between the blepharoplasts of mosses and liverworts, and comparisons with hornworts, biflagellate lycopods and charophytes: A numerical analysis. New Phytology, 117, 187-208. doi:10.1111/j.1469-8137.1991.tb04900.x

[27] Silva, P.C. and Moe, R.L. (2008) Chlorophyceae. AccessScience, McGraw-Hill Companies, New York.

[28] Pandza, A., Baetens, M., Park, C.H., Au, T., Keyhan, M. and Matin, A. (2000) The G-protein FlhF has a role in polar flagellar placement and general stress response induction in Psuedomonas putida. Molecular Microbiology, 36, 414-423. doi:10.1046/j.1365-2958.2000.01859.x

[29] Kusumoto, A., Shinohara, A., Terashima, H., Kojima, S., Yakushi, T. and Homma, M. (2008) Collaboration of FlhF and FlhG to regulate polar-flagella number and localization in Vibrio alginolyticus. Microbiology, 154, 1390 1399. doi:10.1099/mic.0.2007/012641-0

[30] Kusumoto, A., Nishioka, N., Kojima, S. and Homma, M. (2009) Mutational analysis of the GTP-binding motif of FlhF which regulates the number and placement of the polar flagellum in Vibrio alginolyticus. Journal of Biochemistry, 146, 643-650. doi:10.1093/jb/mvp109

[31] Green, J.C., Kahramanoglou, C., Rahman, A., Pender, A.M., Charbonnel, N. and Fraser G.M. (2009) Recruitment of the earliest component of the bacterial flagellum to the old cell division pole by a membrane-associated signal recognition particle family GTP-binding protein. Journal of Molecular Biology, 391, 679-690. doi:10.1016/j.jmb.2009.05.075

[32] Spagnolie, S.E. and Lauga, E. (2011) Comparative hydrodynamics of bacterial polymorphism. Physical Review Letters, 106, Article ID: 058103. doi:10.1103/PhysRevLett.106.058103

[33] Abreu, F., Martins, J.L., Silveira, T.S., Keim, C.N., Lins, de Barros, H.G.P., Filho, F.G.P. and Lins, U. (2007) Candidatus Magnetoglobus multicellularis, a multicellular, magnetotactic prokaryote from a hypersaline environment. International Journal of Systematic and Evolutionary Microbiology, 57, 1318-1322. doi:10.1099/ijs.0.64857-0

[34] Miller, S.M. (2010) Volvox, chlamydomonas, and the evolution of multicellularity. Nature Education, 3, 65. 